\title{
Las salvaguardias, la recaudación fiscal y las importaciones en Ecuador, año 2013 - 2015.
}

Safeguards, tax collection and imports into Ecuador, year 2013 - 2015.

Rivera Vargas José Sebastián. ${ }^{1}$, Vargas Ramos María Fernanda. ${ }^{2}$, Preciado Ramírez Joffre Danny. ${ }^{3}$ \& Cavero Álvarez Omar Damián. ${ }^{4}$

DOI: $\underline{\text { https://doi.org/10.33262/visionariodigital.v2i1.35 }}$

\section{Resumen.}

En el presente documento se analizó el impacto de las salvaguardias en la República del Ecuador con respecto a la Recaudación Fiscal y a las Importaciones tomando los datos de las variables mencionadas según los años 2013 al 2015.

Para realizar el análisis se contextualizó varios conceptos relativos al tema central, posteriormente se realizaron tablas y gráficos para interpretar el comportamiento de las variables mencionadas y revisar el efecto de las Salvaguardias en el proceder de la recaudación del Estado y sus importaciones.

Además, se realizó un modelo econométrico para observar la relación entre las variables, obteniendo un análisis comparativo de los datos en los periodos mencionados con un modelo de regresión simple en el software Gretl.

El Estado Ecuatoriano implementa las salvaguardias como tema de proteccionismo en la producción local y remplazar a la demanda de ciertos productos importados, en cierta forma tomando un efecto positivo para las arcas del Estado.

Palabras clave: Salvaguardias, recaudación fiscal, importaciones, análisis, comportamiento.

\section{Abstract.}

${ }^{1}$ Docente, Universidad Técnica de Ambato, Ambato, Ecuador, j-sebastianrv@ hotmail.com

${ }^{2}$ Docente, Universidad Técnica de Ambato, Ambato, Ecuador, mafervargas7@ 9 hotmail.com

${ }^{3}$ Docente, Universidad Técnica de Ambato, Ambato, Ecuador,danny_ecovs@ @otmail.com

${ }^{4}$ Docente, Universidad Técnica de Ambato, Ambato, Ecuador, omarodca @ hotmail.com 
WwW.visionariodigital.org

This document will analyze the impact of the safeguards in the Republic of Ecuador with respect to the Tax Collection and Imports by taking the data of the mentioned variables following the years: 2013 to 2015.

In order to perform the analysis, several concepts related to the central theme were contextualized, also tables and graphs were then made to interpret the behavior of the mentioned variables and to review the effect of the Safeguards in the proceeding of the collection of the State and its imports.

Keywords: Salvaguards, tax collection, imports, analyze, behavior.

\section{Introducción.}

El estudio económico en el área del ingreso fiscal de los Estados, permite analizar de manera detenida el acontecer de las Naciones, específicamente, el contexto y desarrollo de sus economías.

Es de vital importancia resaltar las decisiones económicas que los gobiernos, ante factores endógenos y exógenos, deben tomar para equilibrar su recaudación fiscal y otras magnitudes de su economía; por ejemplo, las importaciones.

En el caso específico de la República del Ecuador se analizó su toma de decisiones frente a los antecedentes de su economía. Ecuador cuenta con moneda extranjera, los dólares norteamericanos, medida que fue tomada en el año 2000. Dada esta variable, su modelo, estructura política y económica debió cambiar, de cierto modo, drásticamente.

Posterior a los ciclos políticos gubernamentales y económicos se reforma el sistema de recaudación fiscal, aprovechando del mismo, incrementos en los tributos receptados por el Estado, tributos traducidos en impuestos. Ecuador como ya se mencionó cuenta con moneda extranjera la misma que está a la disposición de cambios ya sea en valoración o devaluación, durante el año 2015 ante un incremento del valor del dólar con respecto a otras monedas del mercado internacional, Ecuador se ve afectado en sus exportaciones, pues naciones con productos y calidad similar ofertan a menores precios (con monedas propias) sus bienes en el mercado internacional. En vista de que el crecimiento económico del país requiere una balanza comercial positiva, el Gobierno Nacional en el año 2015 implementa las salvaguardias, valores arancelarios aplicados a las importaciones de ciertos bienes.

Tomando en cuenta este antecedente, se analizará las salvaguardias, posibles efectos, la recaudación fiscal y las importaciones en la República del Ecuador.

\section{Marco teórico referencial.}

\section{Impuestos.}

(Moya Millán, 2001, pág. 191) indica que el impuesto es "como las prestaciones en dinero o en especies, exigidas por el Estado en virtud de su poder de imperio, a quienes se hallen en las situaciones consideradas por la ley como hecho imponible." 
El Impuesto al Valor Agregado es un tipo de rubro a las ventas, medidas por la cantidad de ingresos netos. Pero hay, además, una corrección en esta base de medición, que consiste en restar de los ingresos netos de cada ente económico los costos de los insumos ya gravados adquiridos a otros entes de la economía. Esta deducción nos da como resultado el valor agregado en la etapa correspondiente.

\section{Variación de los impuestos.}

Según (Clavijo, 2000.) una gran herramienta de la política fiscal es el nivel de tributación. Cuando el gobierno de un país baja los impuestos, aumentan los ingresos netos de los hogares. Éstos ahorran parte de esta renta adicional, pero también gastan alguna en bienes de consumo.

Como la reducción de los impuestos eleva el gasto de consumo, desplaza la curva de demanda agregada hacia la derecha. Asimismo, una subida de los impuestos reduce el gasto de consumo y desplaza la curva de demanda agregada hacia la izquierda. En la magnitud del desplazamiento de la demanda agregada provocado por una modificación de los impuestos también influyen el efecto multiplicador y el efecto-expulsión. Cuando el gobierno baja los impuestos y estimula el gasto de consumo, los ingresos y los beneficios aumentan, lo que estimula aún más el gasto de consumo. Éste es el efecto multiplicador. Al mismo tiempo, un aumento de la renta eleva la demanda de dinero, lo que tiende a elevar los tipos de interés.

\section{Decisiones económicas afectadas por los impuestos.}

Los efectos de los impuestos sobre la renta:

(Parkin, Esquivel, \& Muñoz, 2017)explica "el impuesto sobre el ingreso del trabajo influye sobre el PIB potencial y la oferta agregada al cambiar la cantidad de trabajo en pleno empleo. El impuesto sobre la renta debilita el incentivo para trabajar y abre una brecha entre el salario que los trabajadores llevan a sus hogares y los costos de los salarios de las empresas. El resultado es una cantidad más pequeña de trabajo y un PIB potencial más bajo".

Se puede interpretar que este tipo de impuestos crea ineficiencia ya que desincentiva a los trabajadores y por tal motivo existirá factor trabajo inutilizado reduciendo el PIB potencial, el cual es el PIB que se podría alcanzar utilizando todos los factores eficientemente

\section{Multiplicador del impuesto.}

(Parkin, Esquivel, \& Muñoz, 2017) señala "el multiplicador del impuesto es el efecto amplificador de un cambio de los impuestos sobre la demanda agregada. Una disminución de impuestos aumenta el ingreso disponible, lo que a la vez aumenta el gasto de consumo. Una disminución de impuestos funciona como un aumento en las compras gubernamentales".

De acuerdo a este autor se entiende que al reducir los impuestos se puede obtener un efecto favorable ya que se incentiva el consumo lo que dinamiza la economía siendo una medida oportuna en momentos de crisis económicas. 


\section{Importaciones.}

Según Enrique Cornejo Ramírez (1996), define el comercio internacional como “...el intercambio de bienes y servicios entre residentes de diferentes países".

(Krugman, 2012) “...Hay una fuerte relación empírica en un país y el volumen de sus importaciones...”.

Las importaciones son el transporte legítimo de bienes y servicios del extranjero los cuales son adquiridos por un país para distribuirlos en el interior de este. Las importaciones pueden ser cualquier producto o servicio recibido dentro de la frontera de un Estado con propósitos comerciales.

\section{Importaciones en el Ecuador.}

(Simoes, 2016) En 2014 el Ecuador importó \$27,4 miles de millones, lo que es el 69 importador más grande en el mundo. Durante los últimos cinco años las importaciones de Ecuador han aumentado a una tasa anualizada del 12,4\%, de \$15,3 Miles de millones en 2009 a \$27,4 Miles de millones en 2014. Las importaciones más recientes son lideradas por Refinado de Petróleo, que representa el 12,6\% de las importaciones totales de Ecuador, seguido por Alquitrán de aceite, que representa el 8,29\%. (Simoes, 2016)

Tabla 1: Importaciones 2013 - 2015 Ecuador.

\begin{tabular}{cc}
\hline AÑS & VALORES \\
\hline 2013 & $\$ 29.576 .264 .556,46$ \\
2014 & $\$ 30.215 .268 .178,39$ \\
2015 & $\$ 23.933 .474 .181,28$ \\
\hline
\end{tabular}

Fuente: Base de Datos, Banco Mundial (Internet)

Elaborado por: Rivera, Sebastián. Coloma, Marco (2017)

\section{Salvaguardias.}

Las medidas de salvaguardia se definen como medidas "de urgencia" con respecto al aumento de las importaciones de determinados productos cuando esas importaciones hayan causado o amenacen causar un daño grave a la rama de producción nacional del Miembro importador. Esas medidas, que en general adoptan la forma de suspensión de concesiones $\mathrm{u}$ obligaciones, pueden consistir en restricciones cuantitativas de las importaciones o aumentos de los derechos por encima de los tipos consolidados. Constituyen, pues, uno de los tres tipos de medidas especiales de protección del comercio (los otros dos son las medidas antidumping y las medidas compensatorias) a las que pueden recurrir los Miembros de la OMC. 


\section{Implementación de las salvaguardas en Ecuador.}

Comunicado: (Ecuador, 2015) El panorama externo ha modificado las previsiones relacionadas con nuestra balanza de pagos y nos enfrenta a un nuevo escenario que afecta el ámbito comercial como es la baja del precio del petróleo, la apreciación del dólar norteamericano, por lo que se hace necesario tomar medidas para regular el nivel general de las importaciones y equilibrar nuestra Balanza Comercial. Es así que el Gobierno Nacional ha adoptado una serie de medidas para mitigar los impactos de este nuevo escenario, y se ve necesario sustituir la salvaguardia cambiaría por una medida de salvaguardia por balanza de pagos que consiste en la aplicación de aranceles a determinadas importaciones.

Esta medida es aceptada por la Organización Mundial de Comercio (OMC), entidad que permite aplicarla para salvaguardar el equilibrio externo.

La aplicación de esta medida ha sido socializada y debatida con el sector privado, ya que se ha realizado una serie de diálogos y análisis a fin de minimizar el impacto sobre el aparato productivo nacional, tampoco afectaría a mercaderías en tránsito. Los rubros que se han exceptuado de esta medida son:

1. Materias primas y bienes de capital

2. Artículos de higiene personal y uso en el hogar

3. Medicinas y equipo médico

4. Repuestos de vehículos

5. Combustibles y lubricantes

6. Importaciones por correo rápido o courier y menaje de casa

Gráfico 1: Sobretasas y Productos, Salvaguardas.

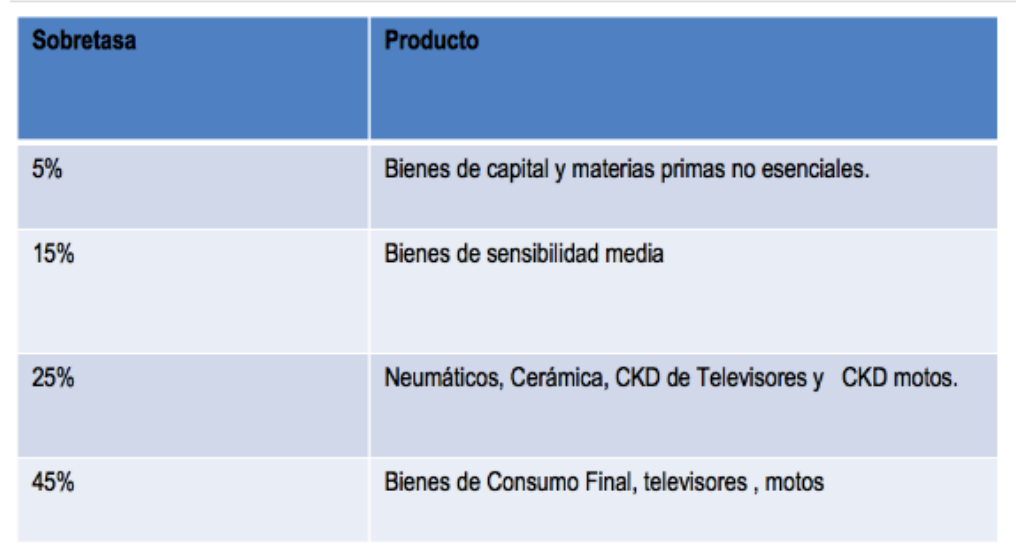

Fuente: Website, Presidencial del Ecuador.

Elaborado por: Website, Presidencial del Ecuador. 


\section{Recaudación fiscal.}

La recaudación fiscal mide la cantidad total de dinero que se consigue con los de impuestos y que será la parte fundamental con la que deberá financiarse los servicios públicos que presta el estado.

\section{Recaudación fiscal en Ecuador.}

El ingreso tributario como proporción del PIB permite analizar los ingresos disponibles en un país recolectados a través de los impuestos con lo cual se financian los bienes y servicios públicos. En este sentido, la comparación regional de este indicador evidencia un incremento considerable de los ingresos tributarios en América Latina que, en 2014, se ubicaron en $21,7 \%$ respecto al PIB regional.

Los aumentos más fuertes los evidenciaron los países de Argentina y Ecuador con incrementos de la participación de los tributos en el PIB de 6,6\% y 4,1\% del PIB respectivamente entre el 2009 y 2014. Este presupuesto traducido en gasto público permite al gobierno ecuatoriano realizar obras para incentivo a la población, en materia de educación, salud, empleo y subempleo.

Tabla 2: Recaudación Fiscal.

\begin{tabular}{ll}
\hline AÑOS & VALORES \\
\hline 2013 & $\$ 12.757 .722,17$ \\
2014 & $\$ 13.616 .817,19$ \\
2015 & $\$ 13.950 .016,01$ \\
\hline
\end{tabular}

Fuente: SRI, (Internet) .

Elaborado por: Rivera, Sebastián. Coloma, Marco (2017).

\section{Metodología.}

Para analizar los posibles efectos de las salvaguardias en la recaudación fiscal y en las importaciones, tomaremos los datos de recaudación del Estado e importaciones de los años, 2013, 2014 y 2015. Tomando en cuenta que a partir del año 2015 se implementan las salvaguardias según decreto Ejecutivo. Se realizará un análisis estadístico mediante cuadros de frecuencia y modelo econometrico mediante el software Gretl.

\section{Recaudación fiscal 2013 - 2015.}


Gráfico 2: Recaudación Fiscal 2013 - 2015 Ecuador.

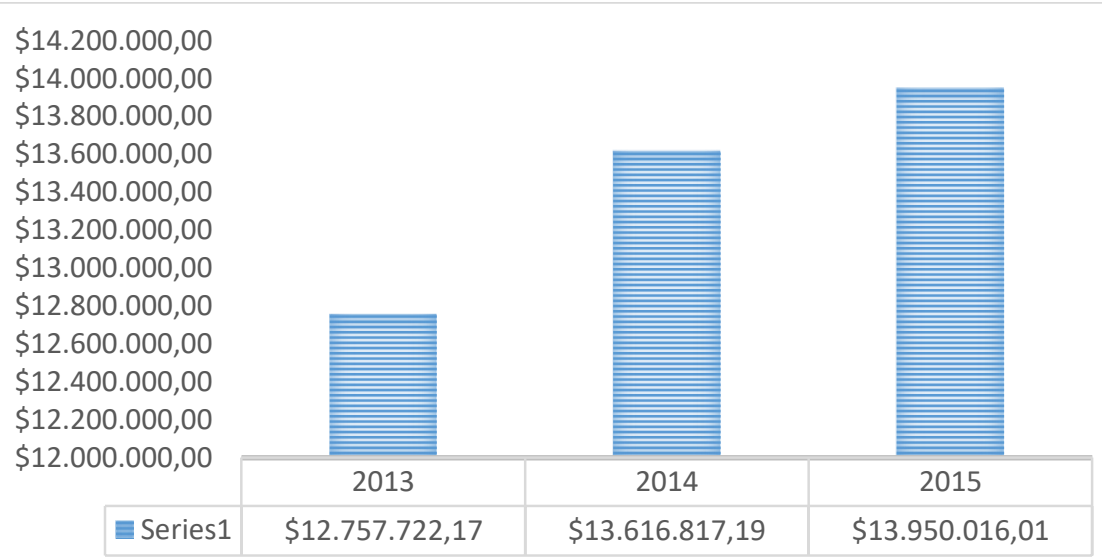

Elaborado por: Grupo de Investigación.

\section{Importaciones.}

Gráfico 3: Importaciones datos 2013 - 2015.

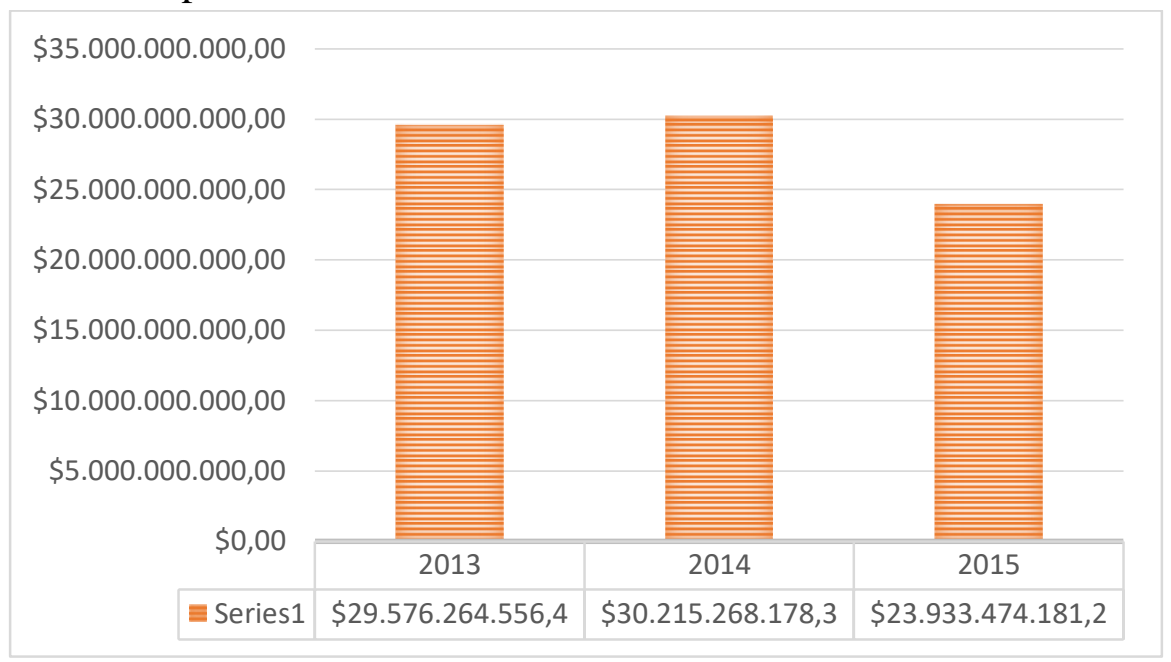

Elaborado por: Grupo de Investigación.

\section{Análisis.}

Recaudación Fiscal:

Según el gráfico 2. El Estado Ecuatoriano en el 2014 ha demostrado un aumento en sus ingresos del 6,73\% con respecto a la recaudación del 2013. En 2015, año de la implementación de las salvaguardia, observamos un crecimiento del 2,45\% con respecto al 2014. En esta ocasión es visible el desincentivo en el consumo y su derivación en el pago de tributos que generan un aumento gradual pero no tan reptante como el de $2013 \mathrm{y}$ 2014. Empezamos a ver un efecto de linealidad a partir de la carga de las salvaguardias al sistema de tributos de Ecuador.

\section{Importaciones:}

Según el gráfico 3. En el año 2014 con respecto del 2013 se observa un aumento de 2,16\% en las importaciones. Las salvaguardias, como medida de protección a la economía 
aprobada por la Organización Mundial de Comercio, (OMC), y adoptadas en el 2015 nos permite apreciar un desincentivo a la importación de bienes en un 2,08\%. Es decir, el efecto de las salvaguardas durante el año de implementación ha sido de reducción.

\section{Análisis de la incidencia de las Importaciones en la Recaudación Fiscal en el período 2013 - 2015.}

Se procederá al análisis a través del software econométrico Gretl, mismo que podemos realizar comparaciones mediante mínimos cuadrados ordinarios, $\mathrm{R}$ cuadrado y las respectivas regresiones. Los datos son obtenidos por el Banco Central del Ecuador en los periodos 2013 al 2015 basándose en miles millones de dólares americanos y se correrá el modelo bajo una regresión lineal simple.

Gráfico 4: Importaciones vs Recaudacion Fiscal datos 2013 - 2015

国 gretl: modelo 1

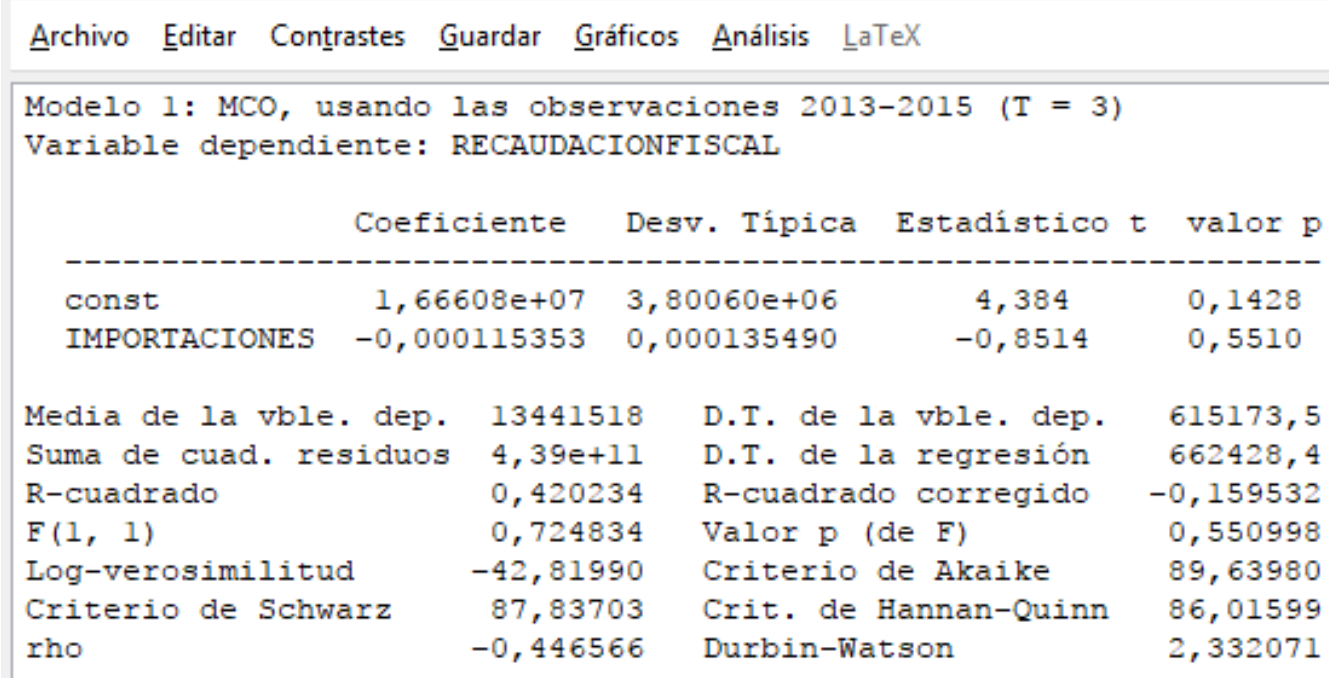

Elaborado por: Grupo de Investigación.

\section{Modelo econométrico.}

\section{Resultados.}

Los calculos obtenidos mediante el software Gretl en la insidencia de las importaciones en la recaudacion fiscal ayudó a obtener un analísis econométrico que permita la estructura en cifras del respectivo modelo quedando de la siguiente manera.

\section{Recaudacion Fiscal = 1,666-0,00011Importaciones $+e$}

Los datos obtenidos por el Banco Central del Ecuador demuestran que el modelo tiene un intercepto del 1,666 y que por cada incremento en las importaciones, la recaudacion fiscal disminuira en - 0,00011 recalcando que existe una mínima reducción en la recaudación fiscal, tomando en cuenta que los valores monetarios de las salvaguardias son ingresos fiscales del estado bajo la presmisa de barrera arancelaria. 
Pese a la redución de las importaciones existe un pequeño aumento en la recadación fiscal tomando en cuenta que los valores monertarios que ingresan a las arcas del estado remplazan a la demanda de ciertos productos importados.

El efecto de las salvaguardias implementado por el Estado es positivo, debido a que las importaciones disminuyeron y pese a su disminucion existe un ligero incremento en su recaudacion fiscal tal como se puede apreciar en el modelo econometrico

\section{Discusión .}

En el año 2015 Chile impone medidas arancelarias a manera de salvaguardias para proteger la producción agrícola específicamente del trigo y la leche, la imposición de medidas en este caso de contingencia para productos agrícolas al mantenerse protegido el sector correspondiente, se termina basando en análisis de ejercicios contra fácticos, como el rechazo del panel del caso de chile - sistema de banda de precios basando este tipo de medidas a "especulaciones y conjeturas",

Es el caso de los productos sujetos a bandas de precio y, en particular, el de la harina de trigo, muestra que, ante la presión de un sector que exhibe una necesidad estructural de protección la institucionalidad chilena tiene suficientes espacios a través de los cuales se puede hacer un uso proteccionista de las medidas de contingencia.

\section{Conclusiones.}

- Las salvaguardias implementadas por el Estado son barreras de protección para la producción local; y una sustitución demanda de ciertos productos importados.

- El efecto de las Salvaguardias implementadas fue negativo en el caso de la Recaudación Fiscal de los años revisados, han incidido en un reducido incremento del ingreso del Estado para el año 2015, sin embargo, no aumentó en el mismo porcentaje del 2014 con respecto del 2013.

- En el caso de las importaciones, el efecto de las salvaguardias fue negativo, produciéndose una reducción de los valores de importación en Ecuador para el año 2015. 


\section{Referencias.}

Clavijo, F. (2000.). “Reformas Económicas en México 1982-1999”. México, FCE,.

Ecuador, P. d. (2015). Sitio Web Presidencia Ecuador. Obtenido de http://www.presidencia.gob.ec/comunicado-oficial-nuevo-sistema-desalvaguardias/

Krugman, P. (2012). Economía Internacional. Pearson.

Moya Millán, E. J. (2001). Elementos de finanzas públicas y derecho tributario . CARACAS, VENEZUELA.

Parkin, M., Esquivel, G., \& Muñoz, M. (2017). “Macroeconomía una versión para Latinoamérica”. México.: Pearson Education.

Simoes, A. (2016). The Observatory of Economic Complexity. Obtenido de http://atlas.media.mit.edu/es/profile/country/ecu/\#Importaciones

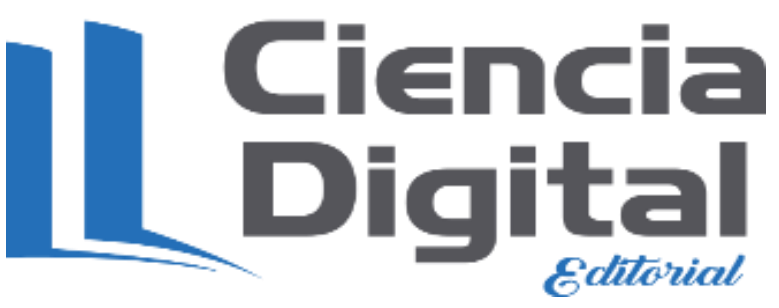




\section{Para citar el artículo indexado.}

Rivera J., Vargas M., Preciado J. \& Cavero O., (2018). Las salvaguardias, la recaudación fiscal y las importaciones en ecuador, año 2013 -2015. Revista electrónica VisionarioDigital1(1), 38-49. Recuperado desde:

http://cienciadigital.org/revistacienciadigital2/index.php/VisionarioDigital/article/view/35/ $\underline{34}$

\section{Ciencia \\ LDigital}

El artículo que se publica es de exclusiva responsabilidad de los autores y no necesariamente reflejan el pensamiento de la Revista Ciencia Digital.

El articulo queda en propiedad de la revista y, por tanto, su publicación parcial y/o total en otro medio tiene que ser autorizado por el director de la Revista Ciencia Digital 